\title{
Developing a Rubric for a Creative CS Principles Lab
}

\author{
Veronica Cateté \\ NC State University \\ 911 Oval Drive \\ Raleigh, NC 27606 \\ vmcatete@ncsu.edu
}

\author{
Erin Snider \\ NC State University \\ 911 Oval Drive \\ Raleigh, NC 27606 \\ eesnider@ncsu.edu
}

\author{
Tiffany Barnes \\ NC State University \\ 911 Oval Drive \\ Raleigh, NC 27606 \\ tmbarnes@ncsu.edu
}

\begin{abstract}
The "Beauty and Joy of Computing" Computer Science Principles class has inspired many new teachers to learn to teach creative computing classes in high schools. However, new computer science teachers feel under-prepared to grade openended programming assignments and support their students' successful learning. Rubrics have widely been used to help teaching assistants grade programs, and are a promising way to support new teachers to learn how to grade BJC programs. In this paper, we adapt general coding criteria from auto-graders to a lab where students write code to draw a brick wall. We tested the rubric on student assignments and showed that we can achieve high inter-rater agreement with the refined rubric.
\end{abstract}

\section{CCS Concepts}

-Social and professional topics $\rightarrow$ K-12 education;

\section{Keywords}

CS Principles, Rubrics, Assessment

\section{INTRODUCTION}

Beauty and Joy of Computing (BJC) is a version of Computer Science Principles (CSP), a new Advanced Placement course, created as an attractive and engaging introduction to computer science that can be taken in high school for college credit. BJC is a rigorous course that uses open-ended Snap! programming problems to teach the seven big ideas of CSP: creativity, abstraction, data and information, algorithms, programming, the internet, and global impact. With funding from the National Science Foundation, the BJC project has provided professional development for over 140 high school teachers nationwide. Teachers enjoy the course and it is well-received by students from diverse backgrounds [8], but teachers report that it is difficult to assess student work and provide students the detailed feedback they need to grow in their computing skills. Most BJC teachers do not

Permission to make digital or hard copies of all or part of this work for personal or classroom use is granted without fee provided that copies are not made or distributed for profit or commercial advantage and that copies bear this notice and the full citation on the first page. Copyrights for components of this work owned by others than ACM must be honored. Abstracting with credit is permitted. To copy otherwise, or republish, to post on servers or to redistribute to lists, requires prior specific permission and/or a fee. Request permissions from permissions@acm.org.

ITiCSE '16, July 09-13, 2016, Arequipa, Peru

(c) 2016 ACM. ISBN 978-1-4503-4231-5/16/07 . \$ $\$ 15.00$

DOI: http://dx.doi.org/10.1145/2899415.2899449 have a computer science background, and therefore have no experience evaluating code, or even in understanding how the complex, open-ended programming labs relate to the seven big ideas of the course. Therefore, it is critical that we help new teachers understand how the tasks students undertake in their labs can be assessed, and how their performance relates to the course's learning objectives.

To address this need, we are defining rubrics for BJC labs that teachers can use to grade student programs. We envision that these rubrics will be usable by new teachers for assessment, and for providing students with meaningful feedback that links to the course's important content. This paper represents the first step in our process - identifying common novice programming grading criteria, adapting them to a specific BJC lab, testing their application on student programs, and refining the rubric to obtain good inter-rater reliability. While grading student programs is straightforward for experienced computer scientists, inexperience with computing and the variety of student code makes this a difficult task for teachers. Less experienced graders must rely on the stated assignment objectives, which can be difficult to translate into numeric grades.

We used a three-phased approach to design and evaluate a rubric for the Brick Wall assignment. In Phase I, we first selected rubric criteria based on literature on automatic support for grading novice programming assignments, and wrote descriptions for 4 levels of performance for each criteria to create an initial rubric. Then, two raters applied the initial rubric to grade 9 student programs independently, and we measured inter-rater reliability. In Phase II, the raters compared their assessments, and refined the rubric and assessments of the initial 9 samples until they agreed upon the scores for each of these projects. Finally, the raters tested the robustness of the revised rubric on 10 additional college assignments, and computed Kappa to measure interrater reliability on the new assignments. We considered a Kappa value of 0.7 or higher to be reliable. In Phase III, we rated all of the projects to compare the results for students taught by an expert high school computer science (CS) teacher with those taught by novice undergraduates.

This work is important in demonstrating that it is possible to create a rubric based on computer science education literature, that can be reliably applied by people with no computing background, to programs that are actually created by novice programmers. Our results show that we can achieve high inter-rater reliability between a computer scientist and a science educator with no computing background, on diverse programs created in labs with teaching assistants 
with no prior programming experience. These results provide strong evidence that we can adapt a general rubric to help novice computing teachers evaluate student programs for particular BJC labs. In the remainder of Section 1, we present the Brick Wall assignment. In Section 2, we explain the development of the Brick Wall rubric. In Section 3, we describe the study methods and phases. In Sections 4 and 5 , we present our results and conclusions.

\subsection{Brick Wall Assignment}

Surveys show that most high school BJC teachers from 2012-2014 used Brick Wall, so we chose it for rubric development. BJC's Brick Wall lab was designed to demonstrate abstraction and the value of creating a function that can be called to perform the same task multiple times, and with different parameters. The main objective of the Brick Wall lab is to create a brick wall with an alternating pattern of bricks. In the assignment, two row types $\mathrm{A}$ and $\mathrm{B}$ are defined, where an A row is made up of whole bricks, and a $\mathrm{B}$ row starts and ends with half-bricks. The assignment specifies that students should create a new block in Snap! that takes 'number of rows' as input and draws a brick wall that alternates A and B type rows accordingly, as shown in Figure 1. Students are instructed to create one method that generates a brick wall, two separate methods that draw the two row types, and a method that draws an individual brick. These specific instructions are explained as levels of abstraction for solving the problem. The visual nature of the task and the clear and simple repetitive structure of a brick wall are affordances that should help make iteration and functions seem to be natural solutions to the problem.

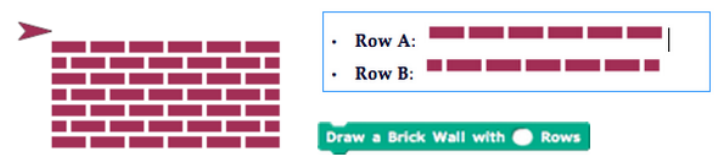

Figure 1: Levels of abstraction for the Brick Wall assignment

\section{RUBRIC CREATION}

To support new teachers to teach computer science, we would ideally build automated systems that could provide both students and teachers with feedback on student performance. Several such intelligent tutoring systems (ITS) have been used to teach programming to novice students. However, the new BJC course strives to inspire new and diverse students to explore computing with modern tools that can be used to express creativity, such as the Snap! programming language. To more quickly support teachers with these new environments, we determined it would be helpful to develop rubrics to help teachers understand the goals of each programming assignment. Our rubric development was strongly influenced by ITSs for programming, since we wish to make an objective system that can be used reliably by teachers with different backgrounds to accurately grade student work. In this section, we describe our process to create a rubric based on computer science education literature. We draw strongly from the literature in intelligent tutoring, where researchers have expended great effort to automate the processes needed to evaluate student code.

\subsection{Rubrics in CS Ed}

In education, rubrics are used as performance-based assessment for student projects. Rubrics use descriptive measures to separate levels of performance on a given task [10]. Then, each level of performance for a particular task is assigned a value. Values in rating scales on rubrics can be either holistic or analytic [2]. Holistic rubrics are quick and efficient, evaluating projects as a whole. Analytic rubrics take longer to design, but offer multiple dimensions that are evaluated separately. Since our goal was to enable teachers to provide more detailed feedback, we chose to design an analytic rubric.

Rubrics can effectively support teaching assistants, who are non-experts in computer science, to quickly, accurately, and consistently assess novice student programs for style, functionality, and design[1]. Researchers tend to agree on three levels of achievement ratings that denote shortcomings, meets expected outcomes, and goes beyond expectations [1, 3, 9]. Stegeman separates expected outcomes into two levels - almost there, and meets expectations [9], and we use this refinement to arrive at four levels. While there is agreement on the levels that programming rubrics should contain, McCauley highlights that it is important that rubrics have precise and consistent language [6]. Toward that end, we chose our rubric criteria from those developed for automated support for grading, as described in the next section.

\subsection{Criteria Selection}

The first step in designing a rubric is to identify a coherent set of criteria for evaluation [2]. When designing our rubric to help teachers evaluate student projects by hand, we wanted to focus on evidence that would be clearly observable in student programs. Since automated systems rely upon objective and observable criteria, they are a natural source for our rubric criteria: Accuracy (correctness), Efficiency (as measured by the number of times blocks are repeated in code), Reasoning (domain knowledge), and Readability (style). These criteria were inspired by Pillay, Zeller, Jackson and Usher as we discuss here.

In 2003, Pillay studied four separate programming tutors and identified four common ways they evaluate student code [7], including: accuracy or correctness of topic, efficiency of the code, adherence to style guidelines, and algorithmic domain knowledge. Domain knowledge is comprised of procedural knowledge on how to write programs and declarative knowledge on how to apply different programming concepts. Since these were common features, we included all four in our initial rubric design.

Jackson and Usher's ASSYST auto-grading system was built to alleviate the errors that people are prone to make when grading, while still keeping a human tutor involved [5]. ASSYST assesses student code for: correctness, efficiency, style, complexity, and test data adequacy. ASSYST measures correctness by how well the student program's output matches the output from a known correct program. Correctness, in BJC, corresponds to whether the program achieves the stated goal, and corresponds to the accuracy metric in Pillay's work and our rubric. ASSYST measures efficiency by keeping track of how often a particular block of code is executed. This assigns higher efficiency to a loop which runs a single command 10 times than code for the same task that uses 10 separate commands each executed once [4]. When 
Table 1: Brick Wall rubric. Additions in italics were refinements to achieve the final rubric.

\begin{tabular}{|l|l|l|l|l|}
\hline Category & 4 & 3 & 2 & 1 \\
\hline Accuracy & $\begin{array}{l}\text { At least 5-6 rows of bricks } \\
\text { created with alternating brick } \\
\text { pattern but all rows same } \\
\text { length }\end{array}$ & $\begin{array}{l}\text { Two rows of brick created } \\
\text { with alternating patterns, or } \\
5-6 \text { inaccurate rows }\end{array}$ & $\begin{array}{l}1 \text { brick created } \\
\text { and repeated }\end{array}$ & 1 brick created \\
\hline Efficiency & $\begin{array}{l}\text { Abstracted methods and } \\
\text { loops used to repeat coding } \\
\text { patterns }\end{array}$ & $\begin{array}{l}\text { A few abstracted methods } \\
\text { were created, but there is still } \\
\text { a lot of repeated code }\end{array}$ & $\begin{array}{l}\text { Code works but } \\
\text { is repeated simple } \\
\text { statements (little } \\
\text { abstraction) }\end{array}$ & $\begin{array}{l}\text { Abstraction of com- } \\
\text { plex methods not } \\
\text { identifiable }\end{array}$ \\
\hline Reasoning & $\begin{array}{l}\text { There is evidence of the use of } \\
\text { mathematical and logical con- } \\
\text { cepts \& appropriate use of ab- } \\
\text { stractions and algorithms. }\end{array}$ & $\begin{array}{l}\text { There is evidence of the use of } \\
\text { mathematical and logical con- } \\
\text { cepts or appropriate use of } \\
\text { abstractions and algorithms. }\end{array}$ & $\begin{array}{l}\text { There is inappro- } \\
\text { priate use of ab- } \\
\text { stractions and al- } \\
\text { gorithms. }\end{array}$ & $\begin{array}{l}\text { Little or no evidence } \\
\text { of the use of math- } \\
\text { ematical and logical } \\
\text { concepts exists }\end{array}$ \\
\hline $\begin{array}{l}\text { The source code has been } \\
\text { commented and the source } \\
\text { code is correct, logical, \& eas- } \\
\text { ily readable. }\end{array}$ & $\begin{array}{l}\text { The source code is correct, } \\
\text { logical, and easily readable. }\end{array}$ & $\begin{array}{l}\text { The source code } \\
\text { is mostly correct, } \\
\text { logical, and read- } \\
\text { able. }\end{array}$ & $\begin{array}{l}\text { The source code is } \\
\text { unclear, incorrect, or } \\
\text { incomplete. }\end{array}$ \\
\hline
\end{tabular}

scoring code by hand, we estimate this by observing whether the projects contain identical code that is written more than once. Repetitive commands in code represent less efficient code, where a loop could have been used instead of copypaste. ASSYST's style metric includes features such as indentation and variable naming. A similar metric can be found in Pillay's work, as well as in Zeller's readability metric, that refers to how easily another person can read and understand how the project code achieves the goals $[7,11]$. We combined these to produce a single Readability criterion for our rubric.

\subsection{Performance Descriptions}

Once we identified the rubric criteria (Accuracy, Efficiency, Reasoning, and Readability), we used the Brick Wall assignment to write descriptions of observable behaviors for each level of performance for each criteria. Brookhart's guidelines suggest that performance level descriptions should be clear, descriptive, and cover the whole range of performance [2]. We started by identifying Level 3 for meets expectations, as described in the assignment. Level 1 descriptions were basically the opposite of these. We developed Level 2 descriptions by identifying how students might have just missed meeting expectations. To develop Level 4 descriptions, we added language about how students might enhance their programs along the lines of the given criteria. Table 1 lists our rubric, with initial descriptions in plain text and the refinements made to create the final rubric added in italics.

\section{STUDY DESIGN AND METHODS}

Our hypothesis was that we could develop a well-defined, analytic rubric, that raters can use to achieve an acceptable level of inter-rater reliability (with Kappa at least 0.7). The following sections describe our the context, data selection, and methods for rating and rubric refinement.

\subsection{Context and Data Sources}

Student code samples for rating were drawn randomly from three classes: two high school elective BJC classes taught by the same teacher in Fall 2013, and one college introductory non-majors computing course taught in Spring 2014. The high school data was from one high school teacher teaching two sections of BJC in Fall 2014. Of the original 42 students, one joined the course halfway through and 2 were missing files, leaving a total of 39 high school submissions. In the Spring 2014 college course with 78 students, 57 files were submitted but 2 were unusable, leaving 55 college submissions. A total of nineteen projects were randomly chosen for analysis, with nine (three from each course) for the initial round, and 10 from the college course for the final round. These projects ranged from completely accurate walls, to one brick telling a joke to a circle.

The two high school classes were taught by an experienced AP Computer Science high school teacher. The college course had lectures by a Computer Science professor, but the seven lab sections were facilitated by five undergraduate teaching assistants (UTAs) with no prior experience in teaching or in BJC. This is similar to the preparation that new BJC high school teachers have, ranging from inexperienced teachers with no computing background to an expert with 20 years of experience teaching advanced computer science in high schools. The five UTA majors were: computer science, civil engineering, materials science, paper science, and textile technology. The UTAs had no prior teaching experience, and no similar computing course. The course professor led the UTAs through the lab the week before students did it, in much the same way that high school teachers keep one step ahead of their students when they teach a new class for the first time. We therefore felt that code from the college course would be representative of work we might expect from students taught by an inexperienced high school teacher with no computing background. Therefore, random samples were chosen from the college course for the evaluation of the final rubric.

\subsection{Methods}

In each phase, two raters worked independently using the rubric to grade each submission, and we computed Kappa for inter-rater reliability at the end of each phase. Rater1 has a Master's in Computer Science, and Rater2 has a PhD in Science Education. In Phase I, both raters rated nine samples from the three courses (three samples from each). To make the selections, we skimmed the programs and determined that the high school submissions were similar, so we selected three from each high school course at random. Since 
we wished to have programs demonstrating diverse performance levels, we made random selections from the college course but continued to make selections until we felt we had samples that were fair, good, and very good.

In Phase II, the raters compared their ratings of all nine samples, and when they did not agree, discussed the ratings, and refined the rubric, as shown in italics in Table 1, until they achieved 100 percent agreement on the ratings for the 9 samples. We will elaborate on some of the contentious coding samples in section 5.2. During this comparison, the raters realized that the quality of submissions from the high school course was higher and less variable. Therefore, the raters selected 10 projects from the college course to test the final, refined rubric for Phase II. As in Phase I, the college projects were selected randomly but samples were excluded if they were very similar to previously-selected samples.

Finally, in Phase III, we trained a third-year undergraduate computer science major to apply the final rubric to the Brick Wall assignment. Rater1 and the new undergraduate (UG) Rater3 applied the final rubric to all 94 Brick Wall assignments, and computed average composite scores for the high school and college projects to compare the performance between the classes. We hypothesized that the experienced high school teacher's students would outperform college students facilitated by university teaching assistants who were inexperienced at both teaching and CS.

\section{RESULTS}

In Phase I, three projects from each section (6 high school, 3 college) were rated using the initial rubric. We found very low agreement between our raters, with a Cohen's Kappa of 0.37. In Phase II, the raters discussed their ratings and refined the rubric, clarifying the descriptions, until they agreed on all ratings for all criteria on the 9 samples. The final rubric is shown in Table 1 with changes between initial and final in italics. In Phase II, the raters applied the resulting final rubric to rate 10 additional college coding samples, and achieved a high level of inter-rater agreement, with Kappa of 0.73. In Phase III, the newly-trained Rater3 achieved a Kappa of 0.735 with Rater 1 and Rater 2 on the 19 projects rated in Phases I and II. Rater 1 and Rater3 then rated the remaining 75 projects and aggregated the rubric scores by high school and college. Results show a clear distinction in performance in labs between the students taught by novices versus the expert high school computer science teacher.

High school students taught by the "master" teacher averaged 3.60 on the Brick Wall assignment. College students taught by novice undergraduates scored an average of 2.79 on the assignment. Figure 2 shows the criteria scores for each class with 25 percent error bars, demonstrating that the high schoolers taught by a master teacher scored quite highly on accuracy and efficiency, and similarly to the college students taught by novices in readability. The largest difference occurs in the reasoning category, where high schoolers were more likely to use conditionals and mathematical expressions. This difference could be due to the teachers, but could also be due to a difference between the self-selection of high school students into a pre-college course, and students taking a low-level computing course as a general analytic elective in college. We present these results to show how the potential differences between novice and master BJC teachers may be impacting student performance in BJC courses.

To illustrate the application of the rubric to student code,

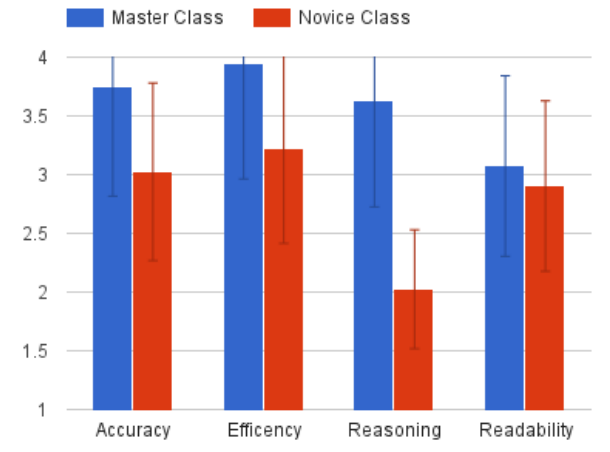

Figure 2: Rubric scores for master and novice teachers

we present sample projects that demonstrate low, medium, or high levels of overall performance, based on standard deviation (SD) away from the overall average for the 19 scored projects. Students more than one SD below average were ranked Low, students one SD above were ranked high. The remaining programs were ranked medium. Of the six high school projects, one was ranked Medium and five were ranked High for demonstrating appropriate abstraction, use of parameters, programming logic and mathematical reasoning. The 13 college class artifacts were rated with two High, seven Medium, and four Low.

\subsection{Code Examples}

In this section, we showcase one example at each performance level (low, medium, and high). Figure 3 shows an example of a low project with the average of all four ratings of 1.75 . The Accuracy score was 3 for creating two rows of bricks with alternating lengths. The Efficiency score was 2, since the code works but consists of repeated simple statements. The Reasoning score was 1 , as there is little or no evidence of the use of mathematical and logical concepts exists. Finally, the Readability score was 1, as the source code is unclear, incorrect, or incomplete.
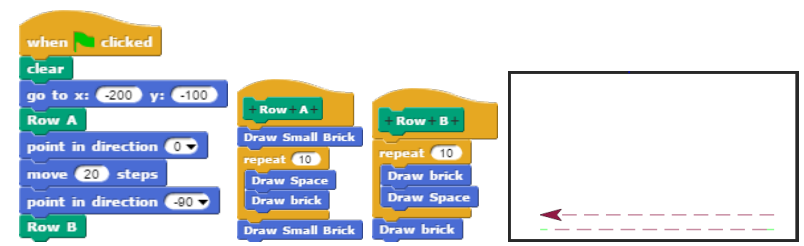

Figure 3: Low-level example, scored as: Accuracy = 3, Efficiency $=2$, Reasoning $=1$, and Readability $=1$.

Figure 4 demonstrates medium performance, with an average score of 3.0. The Accuracy score was 4 for having at least 5-6 rows of bricks with alternating lengths. The Efficiency score was 3 , since a few methods were created, but there is still a lot of repeated code. The Reasoning score was 2 since there is inappropriate use of abstractions and algorithms. Lastly, the Readability scored 3 since the code is correct, logical, and easily readable.

Figure 5 demonstrates high overall performance, with an average score of 3.75. The Accuracy score was 4 since the program creates at least 5-6 rows of bricks with alternating lengths. The Efficiency score was 4 for using methods and 

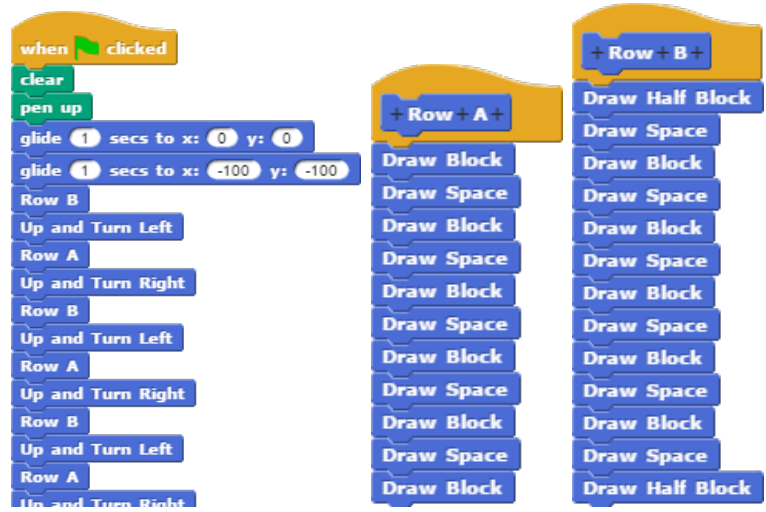

Figure 4: Medium-level example, scored as: Accuracy $=4$, Efficiency $=3$, Reasoning $=2$, and Readability $=3$. This program shows lack of loops and logic

loops to repeat coding patterns. Reasoning also scored 4 since there is evidence of the use of mathematical and logical concepts and appropriate use of abstractions and algorithms. Finally, the Readability scores 3 since the source code is correct, logical, and easily readable.

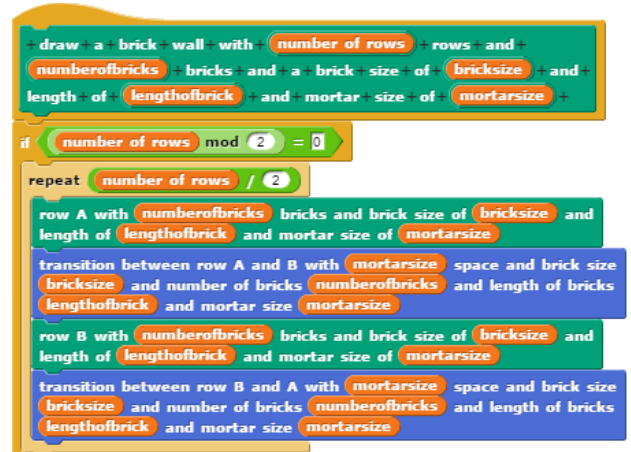

(a) First half of method to generate wall (even \# of rows)

Figure 5: High-level example, scored as: Accuracy $=4$, Efficiency $=4$, Reasoning $=4$, and Readability $=3$. Program uses loops \& math to generate correct output.

These three student code examples demonstrate the diverse ways students can complete an assignment. The low example uses a simple repeat loop, but only creates two rows. The medium example, forgoes loops, rather listing each row separately. The medium and high examples both draw a complete brick wall, but only the high example demonstrates how abstraction and parameters can be used to draw a brick wall customized to user input.

\subsection{Rubric Refinement}

There were four main areas where we edited the initial rubric to resolve rating differences, three in accuracy: misaligned row ends, parameter setting, and code needing edits to run properly; and one in reasoning, with student use of "magic numbers". The Accuracy criterion relies on matching the program's output to the lab assignment's objectives. The lab assignment was generally detailed enough for the two raters to consistently score projects for Accuracy, but a few student programs demonstrated behaviors not accounted for on the rubric. Readability measures how well another person can read someone's code. This category was easily measurable by both raters and adjustments were not required. Efficiency is related to code length - shorter code with blocks that are executed more often than they appear in the program is more efficient. This difference can be seen between 4 which uses the 'Up and Turn Left' and 'Up and Turn Right' blocks each five times, versus 5 which uses each transition block once (it gets repetitively called using a loop). Since this was a straightforward measure, the raters agreed on scores. The Reasoning criterion describes use of logic and abstraction - such as having if or while blocks. The main difference in raters on this was that Rater1 could determine whether mathematical expressions logically matched the program requirements, or if a student inserted a parameter, we call a "magic number" to adjust program output to be correct, despite this number having no basis in logic.

The primary confusion between raters typically revolved around different interpretations of Accuracy. The first difference was in how to score Accuracy for code that correctly draws multiple rows of bricks but whose ends did not align, as shown in Figure 6. Originally, the rubric listed 5-6 rows as a 4 rating and 2 rows as a 3 rating, since we anticipated that some projects would not include loops to create multiple rows. Rater1 scored rows with alternating lengths with a 3 , since they were not entirely accurate, while Rater 2 scored them with a 4 since they had 5-6 rows. Therefore, we added the words "or 5-6 inaccurate rows" to the Accuracy level 3 description to settle this difference.
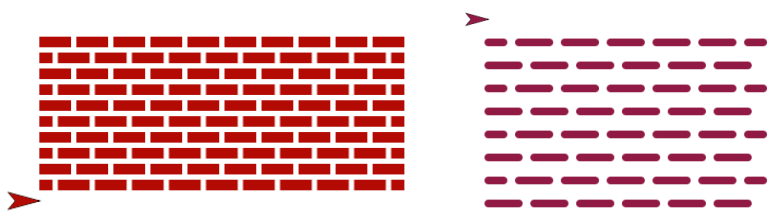

Figure 6: Two brick walls with differing row alignment on the right side.

A second difference between the raters was in judging Accuracy for programs that had adjustable parameters and inputs. In some student projects, the brick wall would not be drawn exactly right unless the rater modified a parameter or input before running the code. Rater1 (computer scientist) scored these higher than Rater2, since it is standard practice in computer science courses to test code with multiple input values. Rater 2 was not familiar with this practice, so rated these projects as incorrect. Upon discussion, the raters agreed to count programs as correct if parameters could be easily adjusted to achieve the correct output, but this was not explicitly added to the rubric.

The raters discovered that some adjustment of the Snap! interface might be needed to fully observe some program behaviors. For a few projects, zooming out or re-centering the stage view made it possible to view the entire wall for evaluation. For a few others, the student had programmed fixed off-screen values for the start and end positions of the wall, relying on the Snap! interface to cut off the wall edges. 
This did not require adjustment of the rubric, but rather adjustment in how the raters used Snap! to run programs.

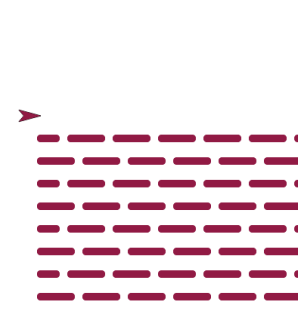

(a) Wall drawn off screen

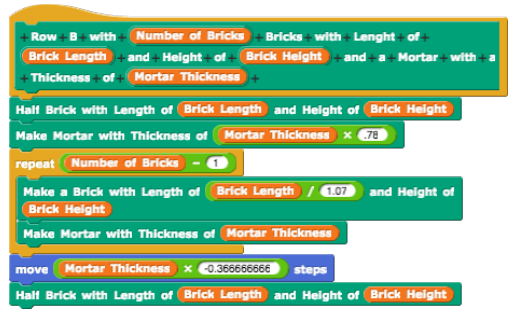

(b) Code shows "magic numbers"

Figure 7: Blocks showing student misunderstandings.

The use of "magic numbers" was the source of confusion for both raters. It was apparent in one example that a student was having difficulty aligning the A and B-type rows and relied upon formulas or numbers to adjust the placement of bricks. However, these "magic numbers" were not applied systematically or in a way that appeared to have a logical reason (to either rater). In these cases, the brick walls looked good but it appeared that students applied a guess and check strategy to determine numbers to use to make the brick wall look correct. The intention of the assignment was for the students to discover a general solution to the brick wall problem, not one that is specific to a particular browser, screen resolution, or idiosyncrasies of student code. In this case, math and logic are clearly applied (since there are mathematical operators visible in the code), but, without a clear formula, it was unclear as to whether or not math and logic were being applied appropriately. This project was also one that had pre-programmed positions for the starting point, and attempts to center the brick wall on stage were difficult to make. It was resolved that there was in fact (inappropriate) use of magic numbers, and another field was added to Reasoning level 2 (Table 1).

\section{CONCLUSIONS AND FUTURE WORK}

In this study, we created an analytic rubric based on evaluation frameworks that automatically assist people in grading student programs, applied the rubric to a set of student work, refined the rubric, and applied it again, demonstrating a dramatic increase in inter-rater reliability. Our rubric rates BJC Brick Wall programs for accuracy, efficiency, reasoning, and readability. In Phase I, we observed that Rater2 (science education) initially had more difficulties with the rubric. In Phase II, after discussion and strategic updates to the rubric, this rater's scores aligned much more closely with those by Rater1 (computer scientist). One limitation of the study is that the raters' discussion likely informed the scoring of assignments in Phase II, so therefore do not represent the likely scores of novice teachers upon first application of the rubric. However, to help address this limitation, we have selected sample student programs that illustrates three levels of proficiency, and will provide these types of samples in our future studies of rubrics as they are used by novice BJC teachers. We also believe that teachers will learn, from the application of the rubric to student code, to differentiate programs according to the rubric criteria, promoting their learning of computer science and their skill in evaluating student programs. Eventually, we believe teachers can use the rubrics to guide students to create better programs.

Another limitation of this study was that the Brick Wall lab is relatively simple. This may be the reason that only minor changes were needed to achieve an acceptable rubric. For more complex assignments, it may not be as straightforward to map the assignment to the rubric criteria. We expect that the readability and efficiency criteria will not need much refinement, but accuracy criteria may need to be added for each program objective, and reasoning may also need individual criteria for each distinct objective.

In future work, we plan to align our rubric with essential knowledge components from the CS Principles course framework available at apcsprinciples.org. For example, Essential Knowledge component 4.1.1 D enumerates the learning goals for Mathematics; specifying iteration, loops, and using the modulus operator. Raters considered similar ideas within the Reasoning category of our rubric, but we could revise the rubric to be more focused on learning objectives, and this is better for providing students target learning goals [2]. By expanding the Reasoning section of our Brick Wall rubric to focus on learning goals and not just task completion, teachers will also be able to better identify what students should be learning and doing during a particular activity or lab.

\section{REFERENCES}

[1] K. Becker. Grading programming assignments using rubrics. In ACM SIGCSE Bulletin, volume 35, pages 253-253. ACM, 2003.

[2] S. M. Brookhart. How to create and use rubrics for formative assessment and grading. ASCD, 2013.

[3] S. Fitzgerald, B. Hanks, R. Lister, R. McCauley, and L. Murphy. What are we thinking when we grade programs? In Proceeding of the 44th ACM technical symposium on Computer science education, pages 471-476. ACM, 2013.

[4] A. Hicks. Bots: Harnessing player data and player effort to create and evaluate levels in a serious game. In Educational Data Mining 2013, 2013.

[5] D. Jackson and M. Usher. Grading student programs using assyst. SIGCSE Bull., 29(1):335-339, Mar. 1997.

[6] R. McCauley. Rubrics as assessment guides. ACM SIGCSE Bulletin, 35(4):17-18, 2003.

[7] N. Pillay. Developing intelligent programming tutors for novice programmers. ACM SIGCSE Bulletin, 35(2):78-82, 2003.

[8] T. W. Price, J. Albert, V. Cateté, and T. Barnes. BJC in Action : Comparison of Student Perceptions of a Computer Science Principles Course. In Proc. of the 1st Annual RESPECT Conference, 2015.

[9] M. Stegeman, E. Barendsen, and S. Smetsers. Towards an empirically validated model for assessment of code quality. In Proceedings of the 14th Koli Calling International Conference on Computing Education Research, pages 99-108. ACM, 2014.

[10] D. D. Stevens and A. J. Levi. Introduction to rubrics. Sterling, VA: Stylus, 2005.

[11] A. Zeller. Making students read and review code. SIGCSE Bull., 32(3):89-92, July 2000. 